\title{
Unterstellte Leseschaften Einleitung
}

Erschienen in: Unterstellte Leseschaften

Von: Hanna Engelmeier, Moritz Baßler

Die Debatten zur Gegenwartsliteratur haben in den letzten Jahren etwas Grundsätzliches bekommen. Bereits eine nur vorläufige Liste von Anlässen, die ein mehr oder weniger breites Echo nicht nur in den sozialen Medien, sondern auch im Feuilleton, in Radio und Fernsehen und bis in die Universität hinein gefunden haben und finden, zeigt: Hier geht es nicht mehr nur um Literarisch-Ästhetisches, sondern es treten jeweils größere weltanschaulich-ethische Aspekte in den Vordergrund, die mit dem Selbstverständnis bestimmter Stilgemeinschaften zu tun haben. ${ }^{1}$ Entsprechend groß ist jeweils das Polarisierungspotential.

Wir denken dabei beispielsweise an die Verwerfungen, die die Erklärung der USLiteraturprofessorin Amy Hungerford ausgelöst hat, sie würde sich fortan weigern, die dicken Romane von David Foster Wallace zu lesen (2016), der bisher als Gegenwartsklassiker galt. ${ }^{2}$ Hierzulande führte die Debatte um das Avenidas-Gedicht von Eugen Gomringer an der Alice-Salomon-Hochschule in Berlin 2017/18 zu hunderten von Stellungnahmen in allen nur denkbaren Medien. ${ }^{3} 2019$ bewirkte der Streit um Takis Würgers Stella beinahe ein Schisma im deutsch-sprachigen Literaturbetrieb. Alle literarischen Instanzen bis hin zu den Buchhändler*innen bezogen dabei massiv Position. ${ }^{4}$ Diskussionsanlässe für sehr basale Fragen zum Status unserer Gegenwartsliteratur boten auch Karen Köhlers Miroloi, ${ }^{5}$ Ann Cottens Lyophilia, ${ }^{6}$ die Nobelpreisverleihungen an Peter Handke (2019) und Louise Glück (2020) sowie die Rezeption von Jasmin Schreibers Marianengraben. ${ }^{7}$ Ein prominentes internationales Beispiel ist die Debatte um Jasmin Cummins' Bestseller American Dirt. ${ }^{8}$ Inzwischen finden sich auf Twitter oder im Feuilleton in regelmäßiger Folge ähnliche Beispiele.

So unterschiedlich die Fälle auf den ersten Blick jeweils gelagert sein mögen - überall begegnet man in den Debatten einer grundlegenden Unklarheit darüber, auf welches Publikum Literatur eigentlich zielt bzw. zielen soll und welches sie tatsächlich erreicht und damit verbunden eine Unklarheit über die literaturästhetischen und -ethischen Maßstäbe. Dem möchten wir in einer Reihe von Beiträgen nachgehen, die zugleich die Konferenz „Unterstellte Leseschaften“ dokumentiert, die im vergangenen September am KWI stattfand. Organisiert wurde diese Tagung von Moritz Baßler, Hanna Engelmeier und Andrea Geier. Ausgehend von den hier genannten Beispielen diskutierten dabei Literatur- und Kulturwissenschaftler*innen, Verleger*innen, Redakteur*innen und Autor*innen darüber, mit welchem Publikum im literarischen Feld überhaupt gerechnet 
werden kann, und wie sich dieses Feld dadurch verändert, dass es auf diese Frage vielleicht gar keine eindeutige Antwort mehr gibt.

In den kommenden Monaten werden im Takt von jeweils ungefähr drei Wochen jeweils zwei bis drei Texte erscheinen, die zu thematischen Schwerpunkten gruppiert einzelne Aspekte der Diskussion aufgreifen. Auf dem Blog des KWI finden sich dabei jeweils knappe Abstracts. Die Forschungsbeiträge verlinken wir - sie erscheinen als OpenAccess Publikationen im Repositorium der Universitätsbibliothek Duisburg-Essen.

Die Diskussion darüber, welche Leseschaften man bei der Produktion und Rezeption von Gegenwartsliteratur überhaupt unterstellen darf, wird von verschiedenen Akteur*innen mit unterschiedlichen Ansprüchen geführt: Auf der einen Seite steht dabei die Klage über einen Verlust basaler literarischer Qualitäten, ästhetischer Sensibilitäten und über eine generelle Kultur des Midcult (Umberto Eco), auf der anderen die ebenso bedenkenswerte Kritik an elitären Ausschlussgesten - der Literatur selbst, aber auch der akademischen und kritischen Instanzen - sowie an der Fortsetzung obsoleter Gewohnheiten im Kunst- und Literaturbetrieb. Die unterstellten wie die tatsächlichen Leseschaften, die der Buchmarkt bedient, scheinen sich in den Bereichen von Habitus, Gender, Bildung, aber auch von im engeren Sinne ästhetischen Erwartungen und Maßstäben derart ausdifferenziert zu haben, dass die alten Wertungs- und Gatekeeperinstanzen (Literaturkritik im Feuilleton, Literaturwissenschaft an der Universität) an Bedeutung verlieren. An ihre Stelle treten zunehmend LeseCommunities, die ihre Informationen und Wertungen vor allem im Netz austauschen.

Grundlegende Veränderungen dieser Art stellen sich nicht über Nacht ein, sondern deuten sich über längere Zeiträume an - und wiederholen dabei auch Figuren aus traditionsreichen Diskursen um Literatursprache und Publikum, wie sie seit der Spätantike geführt werden. ${ }^{9} \mathrm{Zu}$ den diagnostizierten Veränderungen in Literaturbegriff und -praxis tragen diverse Faktoren bei, die hier nur angedeutet werden können. Zum einen findet Literatur, wie Kultur insgesamt, im Verlauf der letzten Jahrzehnte immer deutlicher in marktwirtschaftlichen Strukturen statt. Auflagen- und Verkaufszahlen, zielgruppenorientierte Werbemöglichkeiten (vulgo: Marketing), publikumswirksame Inszenierung von Autor*innen, ganz generell also Strukturen der Rückkopplung lösen den alten Begriff vom autonomen Kunstwerk auf. Sie geben den Rezipient*innen größere Bedeutung und unterlaufen sowohl die alten, hehren Begriffe von einer emphatisch verstandenen KUNST in Großbuchstaben, die sich im expliziten Gegensatz zur Kulturindustrie definierte, als auch die Bourdieu'sche Unterscheidung von einer Literatur, die auf ökonomisches Kapital zielt, und einer, die sich allein um kulturelles Kapital schert.

Zum anderen wird diese generelle Entwicklung, die man positiv als Demokratisierung der Kultur fassen könnte, unterstützt und beschleunigt durch das Web 2.0 und die sozialen Medien, die zu einer generellen Enthierarchisierung der Publikationsmöglichkeiten geführt haben. Indem die medialen Kanäle nun jeder und jedem zur Verfügung stehen, können sich Stilgemeinschaften untereinander barrierefrei 
über ihre präferierten Objekte verständigen. Professionell ausgebildete Expert*innen für Literatur und Kunst verlieren dadurch einen Teil ihrer Privilegien; ihre traditionellen Gatekeeper-Funktionen bleiben nicht mehr konkurrenzlos und unwidersprochen.

Beides, marktwirtschaftliche und mediale Rückkopplung, fördert die Ausbildung und Ausdifferenzierung von durchaus selbstbewussten „Stilgemeinschaften normalisierten Spektakels" (Jochen Venus). Produktionsseitig wird Gegenwartsliteratur längst auf solche Gemeinschaften hin entworfen und vermarktet, rezeptionsseitig bilden sich, mitunter quer dazu, tatsächliche Leseschaften aus, die oftmals mehr eint und trennt als die Liebe zu einer bestimmten Literatur, von politischen Positionen über Fragen der richtigen Ernährung bis hin zu Lifestyle-Entscheidungen. Fragen danach, mit welchen Leseschaften Produzent*innen von Literatur aller Art rechnen und welche Rezeptionsrepertoires den so imaginierten Rezipient*innen vielleicht einfach so lange unterstellt werden, bis sie diese tatsächlich ausbilden, sind in der Literaturwissenschaft vor rund dreißig Jahren schon einmal gestellt worden. Unter anderem in der Auseinandersetzungen mit literarischen Gattungen in der Sozialgeschichte der deutschen Literatur fanden sich Bemühungen, mehr über die sogenannten „Erwartungserwartungen“ des Publikums und von Autor*innen herauszufinden, um so einen Schlüssel zur Erforschung der Entwicklung literarischer Formen zu erhalten. ${ }^{10}$ Diese Diskussion muss heute insbesondere mit Blick auf digitale Lesekulturen aktualisiert werden, wozu die Aufsätze dieses fluiden Sammelbandes beitragen sollen.

Angesichts dieser Entwicklung wird es höchste Zeit, so will es uns scheinen, die Frage nach den impliziten und den realen Leser*innen und Lektüregemeinschaften unserer Gegenwartsliteratur explizit zu stellen, die Frage nach den unterstellten und tatsächlichen Wertungskategorien und Habitus im Lesepublikum, aber auch in der Literaturkritik und bei den Jurys, bei Verlagen und Buchhandel sowie nicht zuletzt bei den Autor*innen und in den Texten (und Paratexten) selbst. Sie verbindet sich mit der Frage nach der Einheit dieser Literatur - ist die überhaupt noch gegeben, oder gibt es, wie beispielsweise in der Pop-Musik, im Grunde nur noch Sparten? Und schließlich: Was ist der Sitz von Gegenwartsliteratur in einer Lebenspraxis, die durch vielfältigen Mediengebrauch charakterisiert ist? Und welche Funktion kommt dabei den genannten Literaturdebatten zu (man könnte ja auch fragen, warum die so häufig von eher marginalen Texten und so selten von wirklich guten Büchern ausgelöst werden)?

Zur Diskussion stehen dabei die Vielfalt und Reichweite unserer ästhetischen Kategorien und damit Qualität und Gültigkeitsanspruch unserer Geschmacksurteile. Hier gilt es zum einen, genau hinzuschauen, wie sich die einzelnen Fälle in ihrer Komplexität darstellen - dabei sind Philologie und Medienanalyse gefragt. Andererseits kann sich eine Bearbeitung dieses Komplexes aber auch um grundsätzliche Befunde und Urteile nicht ganz herumdrücken, zumindest sofern sie selbst Gegenwartsrelevanz beansprucht. Denn es geht dabei auch immer um eine (Neu-)Bestimmung der eigenen Funktion im Gesamtgefüge der Literatur, ob nun als Autor*in, Lektor*in Verleger*in oder Buchhändler*in, als Leser*in, Kritiker*in oder Wissenschaftler*in.

\section{Lesen Sie in den kommenden Wochen folgende Beiträge:}




\section{Mai 2021}

Simon Sahner: Wer liest was? Die Konstruktion des männlichen Lesers in der Gegenwartsliteratur

Berit Glanz: Memes als Wertungen in den sozialen Medien

\section{Mai 2021}

Ines Barner: Auswählen, bearbeiten, adressieren. Lektorat und Lektüreerwartungen Armin Schäfer: Peter Handkes Fiktionen der Leserschaft

\section{Juni 2021}

Johannes Franzen: Everyone's a critic. Literaturkritik in Zeiten des ästhetischen Plebiszit

Christiane Frohmann: Reelle Leseschaften

Ines Barner im Gespräch mit Martin Mittelmeier und Saša Stanišić

\section{Juni 2021}

Andrea Geier: Kanon-Autorität und informiertes Lesen am Beispiel von Christian Krachts „Imperium“

Dirk Knipphals: Gelesen werden. Illusionen, Erfahrungen und Vermutungen eines Literaturredakteurs

\section{Juli 2021}

Silke Horstkotte: Plurale Gesellschaft, plurale Rezeption? Lektürewege postsäkularer Literatur

Hanna Engelmeier: Was macht Tanja Arnheim eigentlich beruflich?

\section{References}

1. Den Begriff übernehmen wir von Jochen Venus, der ihn unter anderem hier entwickelt hat: Jochen Venus: Ausnahme Pop. Über die Unwahrscheinlichkeit einer besonderen ästhetischen Erfahrung, in: LiLi (2016) 46, S. 333-347. https://doi.org/10.1007/s41244-016-0026-2.

2. Siehe: Amy Hungerford: „On not reading«, 16. März 2016, https://www.chronicle.com/article/on-not-readingl.

3. Eine Zusammenfassung der Debatte findet sich u.a. bei Thomas Loer: „Das Gedicht an der Wand «, in: Sozialer Sinn (2019) 19(1), S. 191-226. https://doi.org/10.1515/sosi-2018-0008. 
4. Ausgangspunkt war hier eine gemeinsam verfasste Erklärung von Buchhändler*innen, die den Autor Würger vor einer ihrer Meinung nach unfairen und elitistischen Literaturkritik in Schutz nehmen wollten. In diesem Kommentar von David Hugendick finden sich viele Links zu den in diesem Zusammenhang relevanten Beiträgen: „Was sich verkauft, muss nicht gut sein«, in: ZEIT Online, 4. März 2019, https://www.zeit.de/kultur/literatur/2019-03/takis-wuerger-stellabuchhaendler-literaturkritik-debatte/

5. Moritz Baßler: »Schönheit, Stil und Geschmack«, in: die tageszeitung, 19. August 2019, https://taz.de/Neue-Massstaebe-der-Gegenwartsliteratur/!5615852/, letzter Zugriff 19. April 2021.

6. Hanna Engelmeier: „Wer das liest, ist doof«, in: die tageszeitung, 30. April 2019, https://taz.de/Erzaehlband-der-Dichterin-AnnCotten/!5588238\&SuchRahmen=Print/, letzter Zugriff 19. April 2021.

7. Kontrovers wurde in Sozialen Medien insbesondere der Beitrag von Jan Drees diskutiert, der das Buch vor allem als Herausforderung für die Literaturkritik behandelte: „Somit entsteht, Gott sei's geklagt, eine neue Herausforderung für die klassische Literaturkritik, die ihre Reichweite mehr und mehr teilen muss mit anderen Akteuren. Das ebenso wie Eichborn zu Lübbe gehörende Imprint Lyx verzichtet bereits komplett auf Pressearbeit. Interessant wird zu beobachten sein, wie es weitergeht mit „Marianengraben“ und der Kritik. ", in Deutschlandfunk, 21. Februar 2020, https://www.deutschlandfunk.de/debuetroman-von-jasminschreiber-tod-und-twitter.700.de.html?dram:article_id=470818, letzter Zugriff 19. April 2021-

8. In der taz diskutierte Johannes Franzen insbesondere die Frage nach der kulturellen Aneignung: »Das Feuilleton darf nicht kneifen«, https://taz.de/Kontroverse-um-kulturelle-Aneignung/!5679941/, letzter Zugriff 19. April 2021.

9. Prototypisch entwickelt findet sich diese Diskussion bei Erich Auerbach: Die (lateinisch geprägte) Literatursprache wendet sich laut Auerbach seit der Spätantike immer stärker sogenannten »niederen Formen« zu. Dies wird notwendig, weil da sich die Autoren nicht mehr darauf verlassen können, dass das Publikum Kenntnis eines bestimmten Bildungskanons besitzt, der als gemeinsamer Referenzrahmen zum Verständnis von Texten funktioniert (vgl. Erich Auerbach: Literatursprache und Publikum in der lateinischen Spätantike und im Mittelalter. Bern: Francke, 1958). Auch die gegenwärtige Diskussion ist von Sorgen um das Verschwinden eines gemeinsamen sozialen und ästhetischen Bezugssystems geprägt, das es einerseits erlauben würde, weiterhin gemeinsam von den Klassikern zu zehren und andererseits diese in neueren Texten wiederzuentdecken.

10. Wilhelm Voßkamp: Gattungen als literarisch-soziale Institutionen. Zu Problemen sozial- und funktionsgeschichtlich orientierter Gattungstheorie und -historie, in: Walter Hinck (Hg.): Textsortenlehre - Gattungsgeschichte. Heidelberg 1977, S. 27-44. 
SUGGESTED CITATION: Engelmeier, Hanna; Baßler, Moritz: Unterstellte Leseschaften. Einleitung, in: KWI-BLOG,

[https://blog.kulturwissenschaften.de/unterstellte-leseschaften/], 03.05.2021

DOI: https://doi.org/10.37189/kwi-blog/20210503-0830

DuEPublico

Duisburg-Essen Publications online

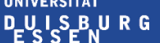

offen im Denken

$\mathbf{U b} \mid \begin{gathered}\text { universitäts } \\ \text { bibliothek }\end{gathered}$

Dieser Text wird via DuEPublico, dem Dokumenten- und Publikationsserver der Universität Duisburg-Essen, zur Verfügung gestellt. Die hier veröffentlichte Version der E-Publikation kann von einer eventuell ebenfalls veröffentlichten Verlagsversion abweichen.

DOI: $\quad$ 10.37189/kwi-blog/20210503-0830

URN: urn:nbn:de:hbz:464-20210503-163854-5

Alle Rechte vorbehalten. 